\title{
Thai Curcuma Species: Antioxidant and Bioactive Compounds
}

\author{
Supawadee Burapan ${ }^{1}$, Mihyang Kim ${ }^{2}$, Yingyong Paisooksantivatana ${ }^{3}$, Bekir Engin Eser ${ }^{4}$ and \\ Jaehong Han $1, * \mathbb{D}$ \\ 1 Metalloenzyme Research Group and Department of Plant Science and Technology, Chung-Ang University, \\ Anseong 17546, Korea; i_chi60@yahoo.com \\ 2 Phytobean, AC. Ltd., Pori 2-gil 16-5, Gamcheon-myeon, Yechon 36810, Korea; mihcoterie@gmail.com \\ 3 Department of Horticulture, Faculty of Agriculture, Kasetsart University, Bangkok 10900, Thailand; \\ yp2624@yahoo.com \\ 4 Department of Engineering, Aarhus University, Gustav Wieds Vej 10, 8000 Aarhus, Denmark; \\ bekireser@eng.au.dk \\ * Correspondence: jaehongh@cau.ac.kr; Tel.: +82-31-670-4830
}

Received: 8 August 2020; Accepted: 1 September 2020; Published: 2 September 2020

\begin{abstract}
For the functional food applications, antioxidant properties and the bioactive compounds of the 23 Curcuma species commercially cultivated in Thailand were studied. Total phenolic content and DPPH radical scavenging activity were determined. The concentrations of eight bioactive compounds, including curcumin (1), demethoxycurcumin (2), bisdemethoxycurcumin (3), 1,7-diphenyl-(4E,6E)-4,6-heptadien-3-ol (4), germacrone (5), furanodienone (6), zederone (7), and ar-turmerone (8), were determined from the Curcuma by HPLC. While the total phenolic content of C. longa was highest $(22.3 \pm 2.4 \mathrm{mg} \mathrm{GAE} / \mathrm{g}$, $\mathrm{mg}$ of gallic acid equivalents), $\mathrm{C}$. Wan Na-Natong exhibited the highest DPPH (2,2-diphenyl-1-picryl-hydrazyl-hydrate) radical scavenging activity. Twenty-three Curcuma species showed characteristic distributions of the bioactive compounds, which can be utilized for the identification and authentication of the cultivated Curcuma species. C. longa contained the highest content of curcumin (1) $(304.9 \pm 0.1 \mathrm{mg} / \mathrm{g})$ and C. angustifolia contained the highest content of germacrone (5) $(373.9 \pm 1.1 \mathrm{mg} / \mathrm{g})$. It was noteworthy that 1,7-diphenyl-(4E,6E)-4,6-heptadien-3-ol (4) was found only from C. comosa at a very high concentration $(300.7 \pm 1.4 \mathrm{mg} / \mathrm{g})$. It was concluded that Thai Curcuma species have a great potential for the application of functional foods and ingredients.
\end{abstract}

Keywords: antioxidant; bioactive; Curcuma; curcuminoids; HPLC; sesquiterpenoids; total phenolic content

\section{Introduction}

Turmeric, Curcuma longa, is the only Curcuma species extensively cultivated and traded in the world [1]. Taxonomically, it belongs to the Curcuma genus of the Zingiberaceae family, and is mostly distributed in Asia [2,3]. Various beneficial health-promoting effects of $C$. longa, such as Alzheimer's disease prevention, anti-inflammatory effects, and HIV-1 protease inhibition, were reported $[4,5]$. Curcuminoids and sesquiterpenoids were identified as the major bioactive constituents of C. longa [6]. More than a hundred species of Curcuma are reported worldwide, and about 30 species among them are cultivated and consumed in Thailand as food additives, cosmetics, and traditional medicines [7]. In particular, many of them are used for the treatment of various diseases due to the existence of bioactive compounds. However, bioactive compounds in other Curcuma species, other than C. longa, have never been studied extensively.

To expand the application of Curcuma species as functional foods, we have collected and cultivated 23 Thai Curcuma species widely consumed in Thailand. Total phenolic content and 
antioxidant activity were measured in the ethanol extracts of dried Curcuma rhizomes. For the bioactive compound study, four curcuminoids and four sesquiterpenoids were isolated and used as reference compounds after complete characterizations, because these are popularly studied representative phytochemicals in Curcuma species. In detail, the curcuminoids curcumin (1), demethoxycurcumin (2), and bisdemethoxycurcumin (3) were isolated from C. longa, and the other curcuminoid 1,7-diphenyl-(4E,6E)-4,6-heptadien-3-ol (4) was isolated from C. comosa. The three germacrane-type sesquiterpenoids germacrone (5), furanodienone (6), and zederone (7) were isolated from C. latifolia, and ar-turmerone (8) was isolated from C. zedoaria (Figure 1). The compositions of these representative bioactive compounds in the Curcuma species were also analyzed by HPLC.<smiles>[R]c1cc(/C=C/C(=O)CC(=O)/C=C/c2ccc(O)c([R])c2)ccc1O</smiles>

$\mathrm{R}_{1}=\mathrm{OCH}_{3}, \mathrm{R}_{2}=\mathrm{OCH}_{3}:$ curcumin (1)

$\mathrm{R}_{1}=\mathrm{H}, \mathrm{R}_{2}=\mathrm{OCH}_{3}:$ demethoxycurcumin (2)

$\mathrm{R}_{1}=\mathrm{H}, \mathrm{R}_{2}=\mathrm{H}$ : bisdemethoxycurcumin $(\mathbf{3})$
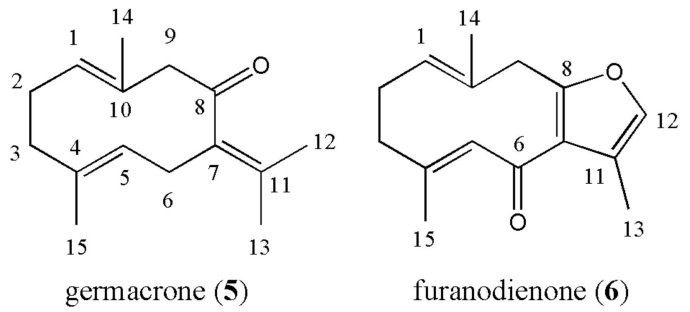

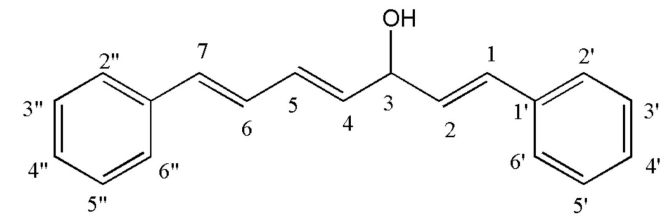

1,7-diphenyl-(4E,6E)-4,6-heptadien-3-ol (4)

Figure 1. Structure of curcuminoids and sesquiterpenoids isolated from Curcuma species.

\section{Materials and Methods}

\subsection{Curcuma Species}

The rhizomes of 23 Curcuma species were collected in Kanchanaburi in 2013 and cultivated in Tapong, Meaung Rayong, Thailand in 2014 throughout the year. The plants that formed flowers were processed to prepare depository specimens at Bangkok Herbarium, Plant Variety Protection Division, Department of Agriculture, Bangkok, Thailand.

\subsection{Plant Materials and Extraction}

Fresh rhizomes of the 23 Curcuma species were rinsed several times with tap water to make them dust and debris free. The rhizomes were then cut into small pieces and dried at room temperature in the shade for 2-3 days. The dried samples were ground to a fine powder and kept in a deep freeze $\left(-70^{\circ} \mathrm{C}\right)$ for further experiments. For extraction, each sample $(1 \mathrm{~g})$ of Curcuma sp. was macerated in $\mathrm{EtOH}(20 \mathrm{~mL})$ for $24 \mathrm{~h}$. The ethanol extract was filtered and dried under reduced pressure. The residue was weighed for the extraction yield and dissolved in $\mathrm{MeOH}$ for the determination of total phenolic content and DPPH radical scavenging assay.

\subsection{Determination of Total Phenolic Contents}

Total phenolic contents of all plant extracts were determined using Folin-Ciocalteu reagent as described by Singleton and Rossi [8]. The plant extracts were dissolved in $\mathrm{MeOH}(1 \mathrm{~mL}, 0.5 \mathrm{mg} / \mathrm{mL})$. Samples were added to microtiter plates and mixed with $100 \mu \mathrm{L}$ of a 10 -fold diluted Folin-Ciocalteu reagent and $80 \mu \mathrm{L}$ of $7.5 \%$ sodium carbonate. The absorbance at $765 \mathrm{~nm}$ was measured using microplate reader spectrophotometers (Spectramax190, Molecular Device, CA, USA) after $30 \mathrm{~min}$. Total phenolic 
content was expressed as mg GAE/g. The calibration curve of standard gallic acid solution was obtained as $y=0.005 x\left(\mathrm{~A}_{765}\right)+0.081, \mathrm{R}^{2}=0.998$, where $y=\mathrm{mg} \mathrm{GAE} / \mathrm{g}$, at the region between 5 and $200 \mu \mathrm{g} / \mathrm{mL}$.

\subsection{DPPH Radical Scavenging Assay}

The antioxidant activity of the Curcuma extracts was evaluated by DPPH radical scavenging. The extract in $\mathrm{MeOH}(2 \mathrm{mg} / \mathrm{mL})$ and ascorbic acid in $\mathrm{MeOH}(200 \mu \mathrm{g} / \mathrm{mL})$ were prepared. The test solution $(50 \mu \mathrm{L})$ was mixed with $950 \mu \mathrm{L}$ of DPPH $(0.1 \mathrm{mM}$ in $\mathrm{MeOH})$. After $30 \mathrm{~min}$, the absorbance at $516 \mathrm{~nm}$ was measured using spectrophotometer (UV/visible detector) in triplicate. DPPH radical scavenging activity was calculated using the following formula: $\%$ scavenging $=100 \times($ (absorbance of control-absorbance of sample test)/absorbance of control).

\subsection{Isolation of Compounds}

The dried rhizomes (500 g) of C. longa were ground and extracted with $\mathrm{EtOH}(1 \mathrm{~L})$ by maceration $(24 \mathrm{~h})$. The dried crude extracts $(5 \mathrm{~g})$ were suspended in water and extracted by EtOAc $(500 \mathrm{~mL}$ $\times 3)$. The combined organic layer $(500 \mathrm{mg})$ was isolated by vacuum liquid chromatography using hexanes and an acetone gradient. Out of the 36 fractions, fractions 25-34 (150 mg) were combined and rechromatographed by column chromatography ( $4 \% \mathrm{MeOH}$ in chloroform) to afford 40 fractions. Curcumin (1) was isolated from fractions 9-14 (30 mg), demethoxycurcumin (2) was isolated from fractions 19-23 (15 mg), and bisdemethoxycurcumin (3) was isolated from fractions 25-35 (55 mg).

Curcumin (1). Yellow powder; ESI $\mathrm{m} / z 369[\mathrm{M}+\mathrm{H}]^{+}$; m.p. $181-183{ }^{\circ} \mathrm{C}$; Anal. calcd for $\mathrm{C}_{21} \mathrm{H}_{20} \mathrm{O}_{6}$ : C 68.47, H 5.47, found: C 67.12, H 5.47. ${ }^{1} \mathrm{H}$ NMR (600 MHz, DMSO- $\left.d_{6}\right): \delta 7.54(2 \mathrm{H}, d, J=15.8 \mathrm{~Hz}, \mathrm{H}-4$, $\left.4^{\prime}\right), 7.32\left(2 \mathrm{H}, d, J=2.0 \mathrm{~Hz}, \mathrm{H}-6,6^{\prime}\right), 7.15\left(2 \mathrm{H}, d d, J=8.3 \mathrm{~Hz}, 2.0 \mathrm{~Hz}, \mathrm{H}-10,10^{\prime}\right), 6.82(2 \mathrm{H}, d, J=8.1 \mathrm{~Hz}$, $\left.\mathrm{H}-9,9^{\prime}\right), 6.75\left(2 \mathrm{H}, d, J=15.8 \mathrm{~Hz}, \mathrm{H}-3,3^{\prime}\right), 6.06(1 \mathrm{H}, \mathrm{s}, \mathrm{H}-1), 3.84\left(6 \mathrm{H}, \mathrm{s}, 7-\mathrm{OCH}_{3}, 7^{\prime}-\mathrm{OCH}_{3}\right) .{ }^{13} \mathrm{C} \mathrm{NMR}^{-}$ (151 MHz, DMSO- $\left.d_{6}\right): \delta 183.26\left(\mathrm{C}-2,2^{\prime}\right), 149.43\left(\mathrm{C}-8,8^{\prime}\right), 148.06\left(\mathrm{C}-7,7^{\prime}\right), 140.76\left(\mathrm{C}-4,4^{\prime}\right), 126.37$ (C-5, $\left.5^{\prime}\right), 123.21\left(\mathrm{C}-10,10^{\prime}\right), 121.13\left(\mathrm{C}-3,3^{\prime}\right), 115.76\left(\mathrm{C}-9,9^{\prime}\right), 111.35\left(\mathrm{C}-6,6^{\prime}\right), 100.91(\mathrm{C}-1), 55.76\left(7-\mathrm{OCH}_{3}\right.$, $\left.7^{\prime}-\mathrm{OCH}_{3}\right)$.

Demethoxycurcumin (2). Yellow orange powder; ESI $m / z 339[\mathrm{M}+\mathrm{H}]^{+}$; m.p. $170-171{ }^{\circ} \mathrm{C}$; Anal. calcd for $\mathrm{C}_{20} \mathrm{H}_{18} \mathrm{O}_{5}$ : C 70.99, $\mathrm{H} 5.36$, found: C 68.53, H 5.17. ${ }^{1} \mathrm{H}$ NMR (600 MHz, DMSO- $\left.d_{6}\right): \delta \delta 10.0$ $\left(1 \mathrm{H}\right.$, br s , 8-OH), $7.56(2 \mathrm{H}, d, J=8.7 \mathrm{~Hz}, \mathrm{H}-6,10), 7.57\left(2 \mathrm{H}, d d, J=15.8 \mathrm{~Hz}, 5.3 \mathrm{~Hz}, \mathrm{H}-4,4^{\prime}\right), 7.31(1 \mathrm{H}, d$, $\left.J=2.0 \mathrm{~Hz}, \mathrm{H}-6^{\prime}\right), 7.14\left(1 \mathrm{H}, d d, J=8.2 \mathrm{~Hz}, 2.0 \mathrm{~Hz}, \mathrm{H}-10^{\prime}\right), 6.82\left(3 \mathrm{H}, d m, \mathrm{H}-7,9,9^{\prime}\right), 6.75(\mathrm{H}, d, J=16 \mathrm{~Hz}$, $\left.\mathrm{H}-3^{\prime}\right), 6.69(\mathrm{H}, d, J=16 \mathrm{~Hz}, \mathrm{H}-3), 6.04(1 \mathrm{H}, \mathrm{s}, \mathrm{H}-1), 3.51\left(1 \mathrm{H}, \mathrm{s}, \mathrm{H}-1^{\prime}\right), 3.83\left(3 \mathrm{H}, \mathrm{s}, 7^{\prime}-\mathrm{OCH}_{3}\right) .{ }^{13} \mathrm{C}$ NMR (125 MHz, DMSO-d 6 ): $\delta 183.69$ (C-2), 183.56 (C-2'), 160.25 (C-8), $149.81\left(\mathrm{C}-8^{\prime}\right), 148.43\left(\mathrm{C}-7^{\prime}\right), 141.13$ (C-4'), 140.79 (C-4), 130.76 (C-6, C-10), 126.76 (C-5'), 126.24 (C-5), 123.63 (C-10'), 121.46 (C-3'), 121.24 (C-3), 116.34 (C-7, C-9), 116.12 (C-9'), 111.68 (C-6'), 101.32 (C-1), $56.13(\mathrm{OMe})$.

Bisdemethoxycurcumin (3). Yellow orange crystals; ESI $m / z 309[\mathrm{M}+\mathrm{H}]^{+}$; m.p. 220-221 ${ }^{\circ} \mathrm{C}$; Anal. calcd for $\mathrm{C}_{19} \mathrm{H}_{16} \mathrm{O}_{5}$ : C 74.01, H 5.23, found: C 69.72, H 5.51. ${ }^{1} \mathrm{H}$ NMR (600 MHz, DMSO- $\left.d_{6}\right): \delta 10.1(2 \mathrm{H}$, br s, 8, $\left.8^{\prime}-\mathrm{OH}\right), 7.57\left(4 \mathrm{H}, d d, J=6.6 \mathrm{~Hz}, 2.1 \mathrm{~Hz}, \mathrm{H}-6,6^{\prime}, 10,10^{\prime}\right), 7.54\left(2 \mathrm{H}, d, J=16 \mathrm{~Hz}, \mathrm{H}-4,4^{\prime}\right), 6.82(4 \mathrm{H}$, $\left.d m, J=8.6 \mathrm{~Hz}, \mathrm{H}-7,7^{\prime}, 9,9^{\prime}\right), 6.70\left(2 \mathrm{H}, d, J=16 \mathrm{~Hz}, \mathrm{H}-3,3^{\prime}\right), 6.04(1 \mathrm{H}, \mathrm{s}, \mathrm{H}-1), 3.51\left(1 \mathrm{H}, \mathrm{s}, \mathrm{H}-1^{\prime}\right) .{ }^{13} \mathrm{C}$ NMR (151 MHz, DMSO-d $\left.d_{6}\right): \delta 183.32\left(\mathrm{C}-2,2^{\prime}\right), 159.88$ (C-8, 8'), $140.48\left(\mathrm{C}-4,4^{\prime}\right), 130.44\left(\mathrm{C}-6,6^{\prime}, 10,10^{\prime}\right)$, $125.93\left(\mathrm{C}-5,5^{\prime}\right), 120.90\left(\mathrm{C}-3,3^{\prime}\right), 116.04\left(\mathrm{C}-7,7^{\prime}, 9,9^{\prime}\right), 101.05(\mathrm{C}-1)$.

The dried rhizome (100 g) of C. comosa was ground and macerated with $\mathrm{MeOH}(200 \mathrm{~mL})$ for $24 \mathrm{~h}$. The dried crude extracts $(2 \mathrm{~g})$ were partitioned by EtOAc and water $(300 \mathrm{~mL} \times 3)$. The EtOAc extract (300 mg) was further isolated by vacuum liquid chromatography using hexanes and acetone. Fractions 12-15 gave 4 (48 mg). 1,7-Diphenyl-(4E,6E)-4,6-heptadien-3-ol (4) Pale yellow solid; ESI m/z $247[\mathrm{M}+\mathrm{H}]^{+}$; Anal. calcd for $\mathrm{C}_{19} \mathrm{H}_{18} \mathrm{O}$ : C 86.99, H 6.92, found: C 86.37, H 7.59. ${ }^{1} \mathrm{H}$ NMR $(300 \mathrm{MHz}$, $\left.\mathrm{CDCl}_{3}\right): \delta 7.27\left(2 \mathrm{H}, b r, \mathrm{H}-3^{\prime}, \mathrm{H}-5^{\prime}\right), 6.75(2 \mathrm{H}), 5.83(\mathrm{H}-4), 4.21(1 \mathrm{H}, \mathrm{s}, \mathrm{OH}), 2.71(\mathrm{H}-7)$. 
The sesquiterpenoids were isolated from the ethanol extract of $C$. latifolia by the same procedure as for curcuminoids. Germacrone (5) was isolated from fractions 9-10 (18 mg), furanodienone (6) was isolated from fraction $15(10 \mathrm{mg})$, and zederone (7) was isolated from fractions 20-22 (38 mg).

Germacrone (5). White crystal; EI $m / z 218[\mathrm{M}]^{+}$; m.p. $55-56{ }^{\circ} \mathrm{C}$; Anal. calcd for $\mathrm{C}_{15} \mathrm{H}_{22} \mathrm{O}$ : C 82.52, $\mathrm{H}$ 10.16, found: C 80.81, H 9.83. ${ }^{1} \mathrm{H}$ NMR $\left(600 \mathrm{MHz}, \mathrm{CDCl}_{3}\right): \delta 4.99(1 \mathrm{H}, b r d, J=12.2 \mathrm{~Hz}, \mathrm{H}-1), 4.71$ $(1 \mathrm{H}, b r d, J=11.2 \mathrm{~Hz}, \mathrm{H}-5), 3.41(1 \mathrm{H}, d, J=10.6 \mathrm{~Hz}, \mathrm{H}-9 \mathrm{a}), 2.99-2.83(3 \mathrm{H}, m, \mathrm{H}-6 \mathrm{a}, \mathrm{H}-6 \mathrm{~b}, \mathrm{H}-9 \mathrm{~b}), 2.37(1 \mathrm{H}$, m, H-2a), 2.19-2.05 (3H, m, H-2b, H-3a, H-3b), 1.78 (3H, s, 13- $\left.\mathrm{CH}_{3}\right), 1.73\left(3 \mathrm{H}, \mathrm{s}, 12-\mathrm{CH}_{3}\right), 1.63(3 \mathrm{H}, \mathrm{s}$, 14- $\left.\mathrm{CH}_{3}\right), 1.44\left(3 \mathrm{H}, \mathrm{s}, 15-\mathrm{CH}_{3}\right) .{ }^{13} \mathrm{C}$ NMR $\left(151 \mathrm{MHz}, \mathrm{CDCl}_{3}\right): \delta 207.92(\mathrm{C}-8), 137.25$ (C-11), 135.00 (C-4), 132.68 (C-1), 129.48 (C-7), 126.68 (C-10), 125.38 (C-5), 55.91 (C-9), 38.09 (C-3), 29.23 (C-6), 24.09 (C-2), 22.34 (C-13), 19.90 (C-12), 16.72 (C-14), 15.59 (C-15).

Furanodienone (6). Yellow pale oil; EI $m / z 230[\mathrm{M}]^{+}$; m.p. 84-86 ${ }^{\circ} \mathrm{C}$; Anal. calcd for $\mathrm{C}_{15} \mathrm{H}_{18} \mathrm{O}_{2}$ : C 78.23, H 7.88, found: C 64.84, H 6.91. ${ }^{1} \mathrm{H}$ NMR (600 MHz, $\left.\mathrm{CDCl}_{3}\right): \delta 7.08(1 \mathrm{H}, m, \mathrm{H}-12), 5.81(1 \mathrm{H}$, $m, \mathrm{H}-5), 5.18(1 \mathrm{H}, b r d d, J=11.7 \mathrm{~Hz}, 4.3 \mathrm{~Hz}, \mathrm{H}-1), 3.70(2 \mathrm{H}, b r d, J=22 \mathrm{~Hz}, \mathrm{H}-9), 2.47(1 \mathrm{H}, d t, J=11.4$ $\mathrm{Hz}, 3.6 \mathrm{~Hz}, \mathrm{H}-3 \mathrm{a}), 2.32(1 \mathrm{H}, m, \mathrm{H}-2 \mathrm{a}), 2.18(1 \mathrm{H}, m, \mathrm{H}-2 \mathrm{~b}), 2.13\left(3 \mathrm{H}, d, J=1.2 \mathrm{~Hz}, 13-\mathrm{CH}_{3}\right), 2.00(3 \mathrm{H}, d$, $\left.J=1.2 \mathrm{~Hz}, 15-\mathrm{CH}_{3}\right), 1.89(1 \mathrm{H}, m, \mathrm{H}-3 \mathrm{~b}), 1.31\left(3 \mathrm{H}, \mathrm{bd}, \mathrm{J}=0.7 \mathrm{~Hz}, 14-\mathrm{CH}_{3}\right) .{ }^{13} \mathrm{C}$ NMR $\left(151 \mathrm{MHz}, \mathrm{CDCl}_{3}\right)$ : $\delta 189.81$ (C-6), 156.52 (C-8), 145.79 (C-4), 138.07 (C-12), 135.39 (C-10), 132.44 (C-5), 130.51 (C-1), 123.71 (C-11), 122.18 (C-7), 41.70 (C-3), 40.66 (C-9), 26.44 (C-2), 18.98 (C-15), 15.78 (C-14), 9.55 (C-13).

Zederone (7). White crystal; EI $\mathrm{m} / \mathrm{z} 246[\mathrm{M}]^{+}$; m.p. $149-151^{\circ} \mathrm{C} ;(69.13 \% \mathrm{C}, 7.11 \% \mathrm{H})$ Anal. calcd for $\mathrm{C}_{15} \mathrm{H}_{18} \mathrm{O}_{3}: \mathrm{C} 73.15, \mathrm{H}$ 7.37, found: C 69.13, H 7.11. ${ }^{1} \mathrm{H}$ NMR $\left(600 \mathrm{MHz}, \mathrm{CDCl}_{3}\right): \delta 7.08(1 \mathrm{H}, m, \mathrm{H}-12)$, $5.48(1 \mathrm{H}, m, \mathrm{H}-1), 3.81(1 \mathrm{H}, s, \mathrm{H}-5), 3.75(1 \mathrm{H}, d, J=16.3 \mathrm{~Hz}, \mathrm{H}-9 \mathrm{a}), 3.69(1 \mathrm{H}, d, J=16.3 \mathrm{~Hz}, \mathrm{H}-9 \mathrm{~b}), 2.52$ $(1 \mathrm{H}, m, \mathrm{H}-2 \mathrm{a}), 2.30(1 \mathrm{H}, d t, J=13.1 \mathrm{~Hz}, 3.5 \mathrm{~Hz}, \mathrm{H}-3 \mathrm{a}), 2.26-2.20(1 \mathrm{H}, m, \mathrm{H}-2 \mathrm{~b}), 2.11(3 \mathrm{H}, d, \mathrm{~J}=1.3 \mathrm{~Hz}$, $\left.13-\mathrm{CH}_{3}\right), 1.34\left(3 \mathrm{H}, d, J=0.8 \mathrm{~Hz}, 15-\mathrm{CH}_{3}\right), 1.32-1.25(1 \mathrm{H}, m, \mathrm{H}-3 \mathrm{~b}) .{ }^{13} \mathrm{C}$ NMR $\left(151 \mathrm{MHz}, \mathrm{CDCl}_{3}\right): \delta$ 192.19 (C-6), 157.08 (C-8), 138.05 (C-12), 131.19 (C-1), 131.04 (C-10), 123.24 (C-11), 122.21 (C-7), 66.54 (C-5), 63.96 (C-4), 41.88 (C-9), 37.98 (C-3), 24.64 (C-2), 15.72 (C-14), 15.14 (C-15), 10.26 (C-13).

ar-Turmerone (8) was isolated from C. zedoaira, and $65 \mathrm{mg}$ of 8 was obtained from $100 \mathrm{~g}$ of the dried rhizomes. ar-Turmerone (8). Colorless oil; $\mathrm{EI} m / z 216[\mathrm{M}]^{+}$; Anal. calcd for $\mathrm{C}_{15} \mathrm{H}_{20} \mathrm{O}: \mathrm{C}$ 83.28, $\mathrm{H}$ 9.32, found: C 79.31, $\mathrm{H} 8.82 .{ }^{1} \mathrm{H}$ NMR $\left(600 \mathrm{MHz}, \mathrm{CDCl}_{3}\right): \delta 7.10(4 \mathrm{H}$, aromatic, $\mathrm{H}), 6.02(1 \mathrm{H}, J=1.3 \mathrm{~Hz}), 3.28$ $(1 \mathrm{H}, d d, J=8.1 \mathrm{~Hz}), 2.70(1 \mathrm{H}, d d, J=15.6 \mathrm{~Hz}), 2.60(1 \mathrm{H}, d d, J=15.6 \mathrm{~Hz}), 2.31\left(3 \mathrm{H}, s,-\mathrm{CH}_{3}\right), 2.10(3 \mathrm{H}, d$, $\left.J=1.3 \mathrm{~Hz},-\mathrm{CH}_{3}\right), 1.85\left(3 \mathrm{H}, d, J=1.3 \mathrm{~Hz},-\mathrm{CH}_{3}\right), 1.25\left(3 \mathrm{H}, d, J=1.3 \mathrm{~Hz}, 12-\mathrm{CH}_{3}\right) .{ }^{13} \mathrm{C} \mathrm{NMR}(151 \mathrm{MHz}$, $\left.\mathrm{CDCl}_{3}\right): \delta 199.85,155.06,143.68,135.53,129.10,126.65,124.08,52.68,35.28,27.63,21.98,20.97,20.70$.

\subsection{Validation of HPLC Analysis}

Validation of HPLC analysis was performed using the purified reference compounds. Calibration curves were constructed from the HPLC peak areas of the reference standards, 1-8, versus their concentrations. Linearity was calculated by measuring the eight points of the calibration curve in the range of $0.003125-0.4 \mathrm{mM}(1-3)$ and $0.00625-0.8 \mathrm{mM} \mathrm{(4-8)}$ with triplicate measurements. The limits of detection (LOD) for 1-8 were found at the micromolar level. Demethoxycurcumin (2) and furanodienone (6) showed the lowest LOD $(2.0 \mu \mathrm{M})$ and limits of quantification (LOQ) at $420 \mathrm{~nm}$ and $245 \mathrm{~nm}$, respectively (Table 1). 
Table 1. Validation of HPLC assay of curcuminoids and sesquiterpenoids.

\begin{tabular}{|c|c|c|c|c|c|}
\hline Compounds & Accuracy ${ }^{1}$ & Linearity & $\begin{array}{c}\text { Correlation } \\
\text { Coefficient }\left(\mathbf{R}^{2}\right)\end{array}$ & $\begin{array}{l}\text { LOD } \\
(\mathrm{mM})\end{array}$ & LOQ (mM) \\
\hline Curcumin (1) & $104.8 \pm 7.9$ & $y=56,277,482 x+476,424$ & 0.9951 & 0.0081 & 0.0245 \\
\hline Demethoxycurcumin (2) & $101.0 \pm 7.9$ & $y=50,455,350 x+56,885$ & 0.9997 & 0.0020 & 0.0061 \\
\hline Bisdemethoxycurcumin (3) & $96.5 \pm 16.2$ & $y=54,929,237 x+369,859$ & 0.9964 & 0.0069 & 0.0209 \\
\hline $\begin{array}{l}\text { 1,7-diphenyl-(4E,6E)- } \\
\text { 4,6-heptadien-3-ol (4) }\end{array}$ & $106.9 \pm 11.6$ & $y=28,237,427 x+612,834$ & 0.9963 & 0.0125 & 0.0377 \\
\hline Germacrone (5) & $97.5 \pm 16.6$ & $y=3,051,741 x+22,600$ & 0.9977 & 0.0110 & 0.0333 \\
\hline Furanodienone (6) & $101.9 \pm 6.8$ & $y=8,043,402 x-10,111$ & 0.9999 & 0.0020 & 0.0060 \\
\hline Zederone (7) & $99.9 \pm 5.1$ & $y=5,130,378 x+25,981$ & 0.9999 & 0.0026 & 0.0080 \\
\hline ar-Turmerone (8) & $98.5 \pm 15.0$ & $y=35,723,577 x+423,877$ & 0.9962 & 0.0125 & 0.0379 \\
\hline
\end{tabular}

${ }^{1}$ All values are presented as the mean \pm SD of triplicate determinations.

\subsection{Compositional Analysis of Curcuma Species}

Each sample $(1 \mathrm{~g})$ of the Curcuma species was macerated in EtOH $(20 \mathrm{~mL})$ for $24 \mathrm{~h}$. The extract was filtered and dried under reduced pressure. The dried residue was dissolved in DMF ( $N, N$-dimethylformamide, $1 \mathrm{mg} / 1 \mathrm{~mL}$ ) for the analysis with a Finnigan Surveyor Plus HPLC system. The mobile phase comprised $0.1 \%$ acetic acid in water (solvent $\mathrm{A}$ ) and $0.1 \%$ acetic acid in $\mathrm{MeCN}$ (solvent B). The injection volume for HPLC analysis was $10 \mu \mathrm{L}$ and the flow was $1.0 \mathrm{~mL} / \mathrm{min}$. For the quantitative analysis, UV absorption at $420 \mathrm{~nm}$ was adopted for 1-3, and UV absorption at $245 \mathrm{~nm}$ was adopted for 4-8.

\subsection{Statistical Analyses}

All measurements were performed in triplicate. Data were processed by Microsoft Excel and reported as means \pm standard deviation.

\section{Results and Discussion}

\subsection{Taxonomy of Curcuma Species}

Among the 23 Curcuma species, 11 Curcuma species, such as C. manga, C. aeruginosa, C. comosa, C. aurantiaca, C. aromatic, C. latifolia, C. zedoaria, C. longa, C. parviflora, C. angustifolia, and C. petiolate, were identified by the taxonomist at the Bangkok Herbarium. The other 12 species are new species, and designated by common names in this report (Table 2).

Table 2. Total phenolic content and antioxidant activity of Curcuma species.

\begin{tabular}{lccc}
\hline \multicolumn{1}{c}{ Curcuma Species } & Extraction Yield (\%) & $\begin{array}{c}\text { Total Phenolic Content } \\
\text { (mg GAE/g Dry Weight) }\end{array}$ & $\begin{array}{c}\text { Antioxidant Activity } \\
\text { (\%) }\end{array}$ \\
\hline Curcuma Wan Ma-Leung & $6.5 \pm 0.4$ & $0.9 \pm 0.0$ & $18.2 \pm 0.3$ \\
\hline Curcumamangga & $6.9 \pm 0.3$ & $0.6 \pm 0.3$ & $9.6 \pm 0.5$ \\
\hline Curcuma Wan Ma-Hor & $8.9 \pm 0.2$ & $0.5 \pm 0.1$ & $13.2 \pm 0.5$ \\
\hline $\begin{array}{l}\text { Curcuma Wan } \\
\text { Khamin-Dam }\end{array}$ & $13.5 \pm 0.2$ & $3.9 \pm 0.4$ & $20.4 \pm 0.4$ \\
\hline Curcuma Wan Rang- Jud & $6.3 \pm 0.3$ & $0.4 \pm 0.1$ & $11.2 \pm 0.2$ \\
\hline Curcumaaeruginosa & $8.6 \pm 0.2$ & $3.2 \pm 0.3$ & $21.5 \pm 0.3$ \\
\hline
\end{tabular}


Table 2. Cont.

\begin{tabular}{|c|c|c|c|}
\hline Curcuma Species & Extraction Yield (\%) & $\begin{array}{l}\text { Total Phenolic Content } \\
\text { (mg GAE/g Dry Weight) }\end{array}$ & $\begin{array}{c}\text { Antioxidant Activity } \\
(\%)^{1}\end{array}$ \\
\hline Curcuma comosa & $16.8 \pm 0.3$ & $4.2 \pm 0.1$ & $90.0 \pm 0.3$ \\
\hline $\begin{array}{l}\text { Curcuma Wan } \\
\text { Kanta-Mala }\end{array}$ & $12.0 \pm 1.0$ & $4.8 \pm 0.1$ & $12.7 \pm 0.3$ \\
\hline Curcumaaurantiaca & $11.9 \pm 0.1$ & $1.5 \pm 0.1$ & $15.2 \pm 0.4$ \\
\hline Curcumaaromatica & $18.5 \pm 0.3$ & $11.0 \pm 0.2$ & $20.8 \pm 0.1$ \\
\hline Curcuma latifolia & $20.3 \pm 0.2$ & $12.9 \pm 0.3$ & $58.6 \pm 0.0$ \\
\hline Curcumazedoaria & $8.5 \pm 0.6$ & $9.3 \pm 0.7$ & $65.7 \pm 0.2$ \\
\hline Curcumalonga & $21.6 \pm 0.5$ & $22.3 \pm 2.4$ & $73.9 \pm 0.1$ \\
\hline Curcumaparviflora & $11.4 \pm 0.5$ & $15.3 \pm 1.2$ & $17.5 \pm 0.5$ \\
\hline Curcumaangustifolia & $3.2 \pm 0.3$ & $2.6 \pm 0.3$ & $3.0 \pm 0.2$ \\
\hline Curcuma Wan Khabitong & $11.7 \pm 0.1$ & $4.6 \pm 0.1$ & $17.0 \pm 0.1$ \\
\hline Curcuma Wan Pataba & $5.8 \pm 0.2$ & $3.3 \pm 0.1$ & $44.5 \pm 0.8$ \\
\hline Curcuma Wan Kortong & $8.4 \pm 0.5$ & $3.3 \pm 0.1$ & $10.4 \pm 0.4$ \\
\hline Curcuma Wan Na-Natong & $3.7 \pm 0.2$ & $5.0 \pm 0.1$ & $91.8 \pm 0.6$ \\
\hline Curcumapetiolata & $5.5 \pm 0.1$ & $2.8 \pm 0.4$ & $11.8 \pm 0.4$ \\
\hline $\begin{array}{l}\text { Curcuma Wan } \\
\text { Khamin-Khao-Padtalod }\end{array}$ & $6.2 \pm 0.3$ & $1.9 \pm 0.8$ & $7.7 \pm 0.2$ \\
\hline Curcuma Wan Chai-Dam & $3.9 \pm 0.2$ & $8.9 \pm 0.2$ & $89.8 \pm 0.6$ \\
\hline $\begin{array}{l}\text { Curcuma Wan } \\
\text { Khamintong }\end{array}$ & $8.6 \pm 0.2$ & $7.7 \pm 1.3$ & $33.3 \pm 0.7$ \\
\hline
\end{tabular}

\subsection{Total Phenolic Content and Antioxidant Activity}

Total phenolic contents of the Curcuma species varied from $0.4 \pm 0.1$ to $22.3 \pm 2.4 \mathrm{mg} \mathrm{GAE} / \mathrm{g}$. The rhizomes of $C$. longa contained the highest phenolic contents $(22.3 \pm 2.4 \mathrm{mg} \mathrm{GAE} / \mathrm{g})$, followed by C. parfivlora (15.3 $\pm 1.2 \mathrm{mg} \mathrm{GAE} / \mathrm{g})$ and C. latifolia $(12.9 \pm 0.3 \mathrm{mg} \mathrm{GAE} / \mathrm{g})$. The DPPH radical scavenging activity of Curcuma extracts is also presented in Table 2. Curcuma Wan Na-Natong showed the highest DPPH radical scavenging activity $(91.8 \pm 0.6 \%)$, followed by C. comosa $(90.0 \pm 0.3 \%)$ and Curcuma Wan Chai-Dam $(89.8 \pm 0.6 \%)$. These three species showed very strong antioxidant activity, even stronger than C. longa, and require further study.

From the results, it was clear that total phenolic contents of Curcuma extracts are not necessarily correlated with the antioxidant activity (Figure 2). Among the three most antioxidant Curcuma rhizomes, C. Wan Na-Natong and C. Wan Chai-Dam are the new species that have never been extensively studied, regardless of their local popularity. The correlation chart (Figure 2) shows that the total phenolic content and DPPH radical scavenging antioxidant activity among the Curcuma species are not necessarily linearly correlated. This is believed to be due to different phytochemical compositions of Curcuma species, as discussed below. 


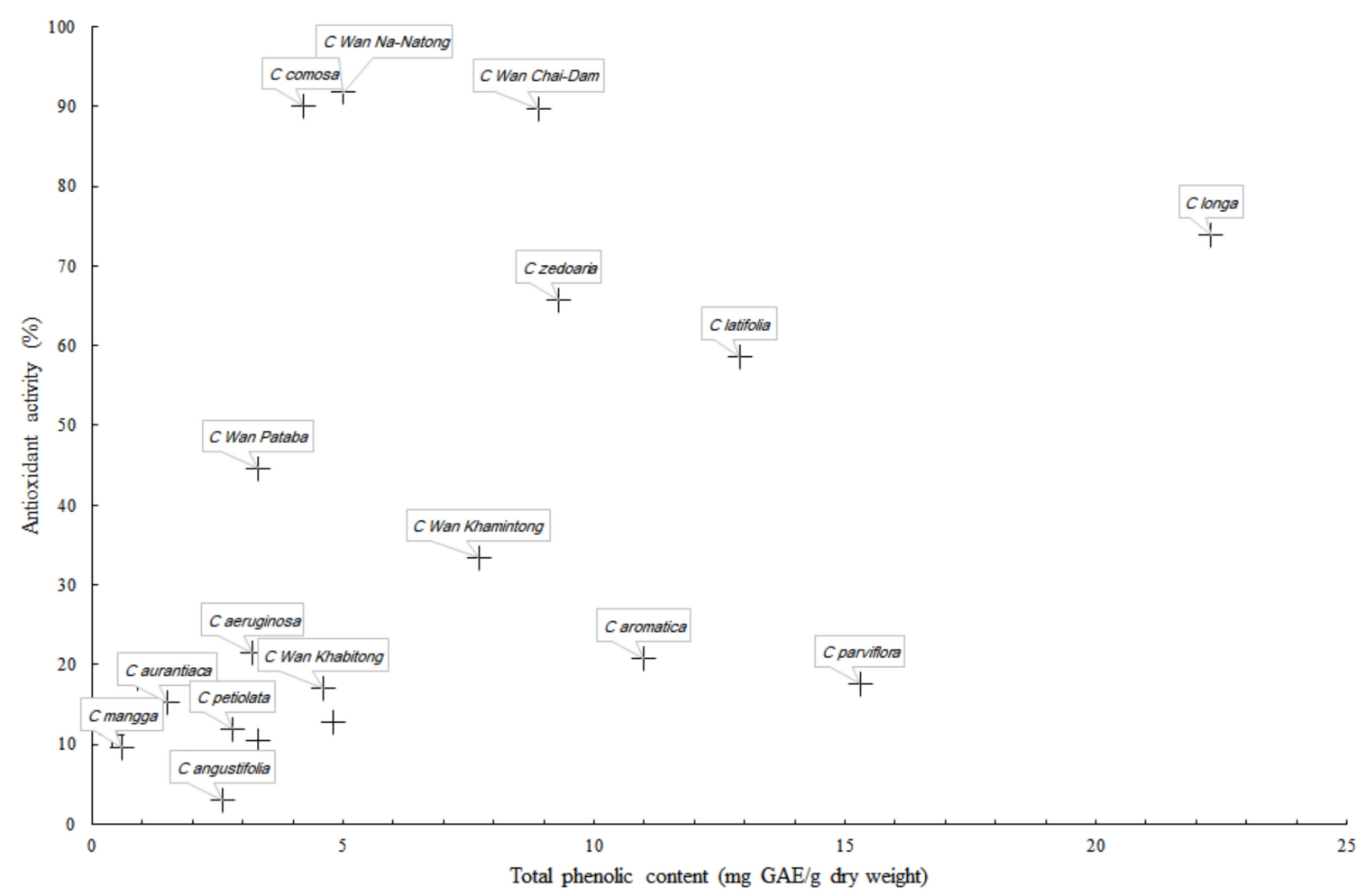

Figure 2. Correlation chart of total phenolic content and DPPH radical scavenging antioxidant activity. The data are from Table 2, and only notable data are labeled in the chart.

\subsection{Isolation of Bioactive Curcuminoids and Sesquiterpenoids}

Curcuminoids 1-3 were isolated from C. longa, and $\mathbf{4}$ was isolated from C. comosa. Sesquiterpenoids 5-7 were isolated from C. latifolia for the first time in this study. Compound $\mathbf{8}$ was isolated from C. zedoaria. The structures of the isolated reference compounds (Figure 1) were confirmed by the comparison of melting point, elemental analysis, ${ }^{1} \mathrm{H}$, and ${ }^{13} \mathrm{C}$ NMR data (see the Supplementary Materials) [9-16].

\subsection{Chemical Composition of 23 Curcuma Species}

Curcuminoids 1-3 are considered as the most characteristic bioactive secondary metabolites found in turmeric. Therefore, we analyzed the collected Curcuma species to find out whether the rhizomes contain 1-3. The EtOH extracts (1 mg/1 mL) were analyzed with HPLC at $420 \mathrm{~nm}[17,18]$, and only ten species were found to contain curcuminoids (Table 3). Furthermore, only three Curcuma species, C. zedoaria, C. longa, and Curcuma Wan Khamintong, contained all three curcuminoids (Figure 3). Trace amounts of curcuminoids were identified from C. manga, Curcuma Wan Rang-Jud, C. comosa, C. latifolia, C. parviflora, Curcuma Wan Pataba, Curcuma Wan Na-Natong, and C. petiolate under LOQ, only when the higher concentrations of the analytes were analyzed by HPLC. It is noticeable that C. longa was found to contain the highest curcuminoid content $(653 \mathrm{mg} / \mathrm{g})$. Even though curcumin (1) is the representative bioactive compound of turmeric, only nine Curcuma species contained curcumin (1). Demethoxycurcumin (2) was the major curcuminoid of four other Curcuma species, C. aeruginosa, C. aurantiaca, C. aromatica, and C. zedoaria. 
Table 3. Analysis of curcuminoids from the Curcuma species.

\begin{tabular}{lccc}
\hline \multicolumn{1}{c}{ Curcuma Species } & $\mathbf{1}$ & $\mathbf{2}$ & $\mathbf{3}$ \\
\hline Curcuma Wan Ma-Leung & $5.2 \pm 0.1$ & $4.1 \pm 0.0$ & $\mathrm{ND}^{1}$ \\
Curcuma Wan Ma-Hor & $\mathrm{ND}$ & $0.2 \pm 0.0$ & $\mathrm{ND}$ \\
Curcuma aeruginosa & $35.5 \pm 0.7$ & $107.2 \pm 1.0$ & $\mathrm{ND}$ \\
Curcumaaurantiaca & $3.2 \pm 0.0$ & $6.5 \pm 0.0$ & $\mathrm{ND}$ \\
Curcuma aromatica & $24.3 \pm 0.1$ & $112.5 \pm 1.0$ & $\mathrm{ND}$ \\
Curcuma zedoaria & $72.3 \pm 0.6$ & $201.5 \pm 1.5$ & $28.2 \pm 0.8$ \\
Curcuma longa & $304.9 \pm 0.1$ & $189.2 \pm 0.4$ & $158.8 \pm 0.7$ \\
Curcuma Wan Khabitong & $10.2 \pm 0.1$ & $6.6 \pm 0.0$ & $\mathrm{ND}$ \\
Curcuma Wan Kortong & $0.1 \pm 0.0$ & $1.8 \pm 0.0$ & $\mathrm{ND}$ \\
Curcuma Wan Khamintong & $47.2 \pm 0.1$ & $37.1 \pm 0.1$ & $8.0 \pm 0.0$ \\
\hline
\end{tabular}

${ }^{1} \mathrm{ND}=$ in trace below detection limit.
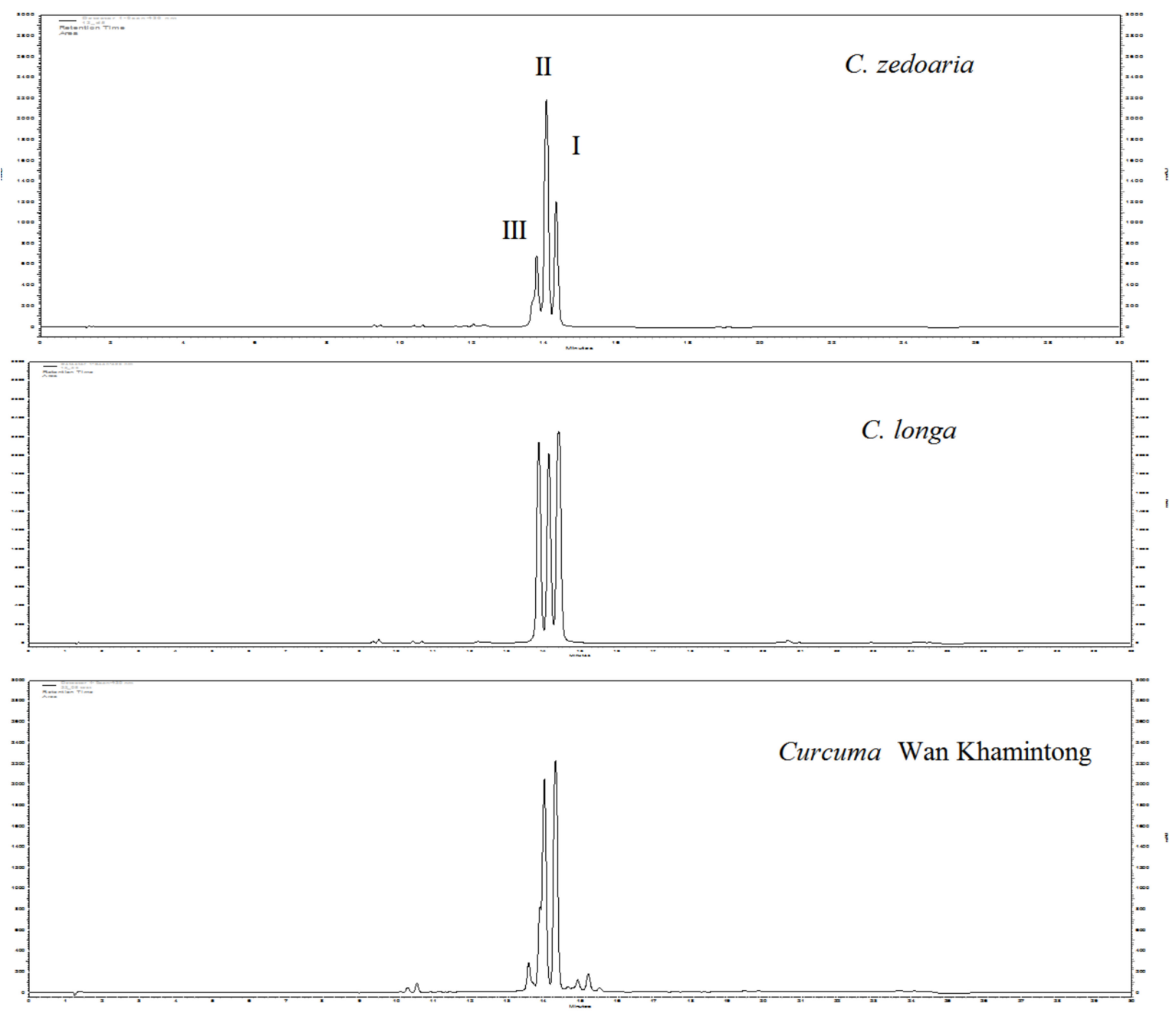

Figure 3. HPLC chromatogram $(420 \mathrm{~nm})$ for C. zedoaria, C. longa, and Curcuma Wan Khamintong; I: curcumin (1), II: demethoxycurcumin (2), III: bisdemethoxycurcumin (3).

From Indonesian Curcuma species, C. mangga, C. heyneana, C. aeruginosa, and C. soloensis were reported to contain curcuminoids [19]. Our analysis also confirmed that C. mangga and C. aeruginosa contained curcuminoids. C. aurantiaca contained high contents of $\mathbf{1}-\mathbf{3}$, and this is the first report of the identification of 1-3 in the rhizomes of $C$. aurantiaca. Diarylheptanoid 1,7-diphenyl-(4E,6E)-4,6-heptadien-3-ol (4) belongs to the curcuminoids, but it was found only from C. comosa in a high content of $300.7 \pm 1.4 \mathrm{mg} / \mathrm{g}$. It is noteworthy that $C$. comosa does not contain 1-3. 
There are more than 100 different sesquiterpenoids reported from Curcuma rhizomes [20], and these secondary bioactive metabolites are also responsible for some of the biological activities observed from the rhizomes of Curcuma species. Therefore, we isolated three germacrane-type sesquiterpenoids, germacrone (5), furanodienone (6), and zederone (7), which is the first report from C. latifolia. In addition, ar-turmerone (8) was isolated from C. zedoaria for the first time. Germacrone (5) is known to exhibit anti-inflammatory [21], antiviral [22], and anti-cancer activities [23], and was found in the various Curcuma species, such as C. aeruginosa, C. amanda, C. aromatica, C. xanthrrhiza, and C. zedoaria.

Among the 23 Curcuma species analyzed, 21 species were found to contain at least one of the four sesquiterpenoids, and only two Curcuma species, C. aurantiaca and C. zedoaria, contained all four sesquiterpenoids (Table 4). Generally, germacrone (5) was the most abundant sesquiterpenoid in the Curcuma species, and C. angustifolia contained only 5, out of the eight reference compounds. In particular, C. angustifolia showed a very high concentration of 5 (37\% of extract), and Curcuma Wan Khamin-Khao-Padtalod contained only ar-turmerone (8).

Table 4. Analysis of sesquiterpenoids from the ethanol extraction residues of Curcuma species.

\begin{tabular}{|c|c|c|c|c|}
\hline \multirow{2}{*}{ Curcuma Species } & \multicolumn{4}{|c|}{ Sesquiterpenoids $(\mathrm{mg} / \mathrm{g})^{1}$} \\
\hline & 5 & 6 & 7 & 8 \\
\hline Curcuma Wan Ma-Leung & $42.6 \pm 0.9$ & $\mathrm{ND}^{2}$ & ND & ND \\
\hline Curcuma Wan Ma-Hor & $47.5 \pm 0.7$ & $4.0 \pm 0.0$ & $6.6 \pm 0.2$ & ND \\
\hline Curcuma Wan Khamin-Dam & $126.5 \pm 0.3$ & $18.5 \pm 0.1$ & ND & ND \\
\hline Curcuma aeruginosa & ND & $3.1 \pm 0.0$ & ND & ND \\
\hline Curcuma comosa & $31.1 \pm 0.2$ & $81.0 \pm 0.9$ & $37.6 \pm 0.2$ & ND \\
\hline Curcuma Wan Kanta-Mala & $106.9 \pm 0.4$ & $15.7 \pm 0.1$ & ND & ND \\
\hline Curcuma aurantiaca & $36.5 \pm 0.4$ & $15.4 \pm 0.5$ & $8.6 \pm 0.1$ & $4.9 \pm 0.0$ \\
\hline Curcuma aromatica & $73.7 \pm 2.8$ & $4.7 \pm 0.0$ & $33.2 \pm 0.6$ & ND \\
\hline Curcuma latifolia & $56.2 \pm 0.3$ & $73.3 \pm 1.1$ & $61.3 \pm 0.1$ & ND \\
\hline Curcuma zedoaria & $7.5 \pm 0.1$ & $15.5 \pm 0.4$ & $3.3 \pm 0.0$ & $53.9 \pm 0.2$ \\
\hline Curcuma longa & ND & ND & ND & $78.4 \pm 0.2$ \\
\hline Curcuma parviflora & ND & $3.6 \pm 0.1$ & ND & ND \\
\hline Curcuma angustifolia & $285.3 \pm 0.6$ & ND & ND & ND \\
\hline Curcuma Wan Khabitong & $6.8 \pm 0.1$ & ND & ND & $4.4 \pm 0.2$ \\
\hline Curcuma Wan Patab & $31.2 \pm 0.3$ & $2.5 \pm 0.0$ & ND & $0.2 \pm 0.0$ \\
\hline Curcuma Wan Kortong & ND & ND & ND & $3.5 \pm 0.2$ \\
\hline Curcuma Wan Na-Natong & $144.3 \pm 2.8$ & $47.1 \pm 0.4$ & $13.2 \pm 0.1$ & ND \\
\hline Curcuma petiolata & ND & ND & ND & $0.4 \pm 0.0$ \\
\hline Curcuma Wan Khamin-Khao-Padtalod & ND & ND & ND & $17.6 \pm 0.1$ \\
\hline Curcuma Wan Chai-Dam & ND & $65.1 \pm 0.2$ & $13.5 \pm 0.3$ & ND \\
\hline Curcuma Wan Khamintong & ND & ND & ND & $4.6 \pm 0.0$ \\
\hline
\end{tabular}

${ }^{1}$ Based on the weight of ethanol extraction residue from dried Curcuma species (wt $\left.\%\right) .{ }^{2} \mathrm{ND}=$ not detected.

\section{Conclusions}

The phytochemical property of 23 Curcuma species was studied by means of total phenolic contents and antioxidant activity assays, as well as compositional analysis. Besides, eight bioactive curcuminoids and sesquiterpenoids were isolated from the rhizomes of C. longa, C. comosa, C. latifolia, and C. zedroaria. In particular, the isolation of germacrone (5), furanodienone (6), and zederone (7) is the first report from C. latifolia. From the compositional analysis, C. longa was distinct in its highest curcumin (1) concentration. C. angustifolia was found to contain the highest content of germacrone (5). Out of 23 Curcuma species, five Curcuma species did not contain any curcuminoids 1-4. None of the analyzed Curcuma species contained all eight bioactive compounds. Therefore, the data of phytochemical profiling can be used for the identification and authentication of cultivated Curcuma species. In addition, there is a tremendous potential for the utilization of less-studied Curcuma species as functional foods and ingredients. 
Supplementary Materials: The following are available online at http://www.mdpi.com/2304-8158/9/9/1219/s1, Table S1: List of 23 Curcuma species, Figure S1: Spectral data for the isolated compounds, Table S2: Taxonomy of 23 Curcuma species.

Author Contributions: Conceptualization, J.H. and Y.P.; methodology, S.B. and M.K.; validation, M.K., B.E.E., and J.H.; investigation, S.B. and M.K.; resources, S.B. and Y.P.; data curation, S.B.; writing-original draft preparation, J.H.; writing-review and editing, B.E.E. and J.H.; visualization, X.X.; supervision, J.H.; funding acquisition, J.H. All authors have read and agreed to the published version of the manuscript.

Funding: This research was supported by the Chung-Ang University Research Grants in 2019 and the framework of the international cooperation program managed by the National Research Foundation of Korea (NRF-2017K2A9A1A01092924).

Acknowledgments: The authors thank the staff at the Bangkok Herbarium, Thailand for the technical support on the taxonomic study of Curcuma species.

Conflicts of Interest: The authors declare no conflict of interest.

\section{References}

1. Ravindran, P.N.; Nirmal, B.K.; Sivaraman, K. (Eds.) Tumeric-The Genus Curcuma; CRC Press: Boca Raton, FL, USA, 2007; p. 504.

2. Leong-Skornickova, J.; Sida, O.; Jarolimova, V.; Sabu, M.; Fer, T.; Travnicek, P.; Suda, J. Chromosome numbers and genome size variation in Indian species of Curcuma (Zingiberaceae). Ann. Bot. Lond. 2007, 100, 505-526. [CrossRef] [PubMed]

3. Chen, J.; Xia, N.; Zhao, J.; Chen, J.; Henny, R. Chromosome numbers and ploidy levels of Chinese Curcuma species. Hortic. Sci. 2013, 48, 525-530. [CrossRef]

4. Burapan, S.; Kim, M.; Han, J. Curcuminoid demethylation as an alternative metabolism by human Intestinal microbiota. J. Agric. Food Chem. 2017, 65, 3305-3310. [CrossRef]

5. Pinkaew, D.; Changtam, C.; Tocharus, C.; Thummayot, S.; Suksamrarn, A.; Tocharus, J. Di-O-demethylcurcumin protects SK-N-SH cells against mitochondrial and endoplasmic reticulum-mediated apoptotic cell death induced by $\mathrm{A} \beta_{25-35}$. Neurochem. Int. 2015, 80, 110-119. [CrossRef]

6. Agarwal, N.B.; Jain, S.; Nagpal, D.; Agarwal, N.K.; Mediratta, P.K.; Sharma, K.K. Liposomal formulation of curcumin attenuates seizures in different experimental models of epilepsy in mice. Fundam. Clin. Pharmacol. 2013, 27, 169-172. [CrossRef]

7. Habsah, M.; Amran, M.; Mackeen, M.M.; Lajis, N.H.; Kikuzaki, H.; Nakatani, N.; Rahman, A.A.; Ali, A.M. Screening of Zingiberaceae extracts for antimicrobial and antioxidant activities. J. Ethnopharmacol. 2000, 72, 403-410. [CrossRef]

8. Singleton, V.L.; Rossi, J.A. Colorimetry of total phenolics with phosphomolybdic-phosphotungstic acid reagents. Am. J. Enol. Viticult. 1965, 16, 144-158.

9. Uehara, S.I.; Yasua, I.; Akiyama, K.; Morita, H.; Takeya, K.; Itokawa, H. Diarylheptanoids from the rhizomes of Curcuma xanthorrhiza and Alpinia officinarum. Chem. Pharm. Bull. 1987, 35, 3298-3304. [CrossRef]

10. Shibuya, H.; Hamamoto, Y.; Cai, Y.; Kitagawa, I. A reinvestigation of the structure of zederone, a furanogermacrane-type sesquiterpene from zedoary. Chem. Pharm. Bull. 1987, 35, 924-927. [CrossRef]

11. Firman, K.; Kinoshita, T.; Itai, A.; Sankawa, U. Terpenoids from Curcuma heyneana. Phytochemistry 1988, 27, 3887-3891. [CrossRef]

12. Dekebo, A.; Dagne, E.; Hansen, L.K.; Gautun, O.R.; Aasen, A.J. Crystal structures of two furanosesquiterpenes from Commiphora sphaerocarpa. Tetrahed. Lett. 2000, 41, 9875-9878. [CrossRef]

13. Barrero, A.F.; Herrador, M.M.; Lopez-Perez, J.; Arteaga, J.F.; Catalan, J. New pathways in transannular cyclization of germacrone [germacra-1(10),4,7(11)-trien-8-one]: Evidence regarding a concerted mechanism. Org. Lett. 2009, 11, 4782-4785. [CrossRef]

14. Sukari, M.A.; Wah, T.S.; Saad, S.M.; Rashid, N.Y.; Rahmani, M.; Lajis, N.H.; Hin, T.Y.Y. Bioactive sesquiterpenes from Curcuma ochrorhiza and Curcuma heyneana. Nat. Prod. Res. 2010, 24, 838-845. [CrossRef] [PubMed]

15. Kader, M.G.; Habib, M.R.; Nikkon, F.; Yeasmin, T.; Rashid, M.A.; Rahman, M.M.; Gibbons, S. Zederone from the rhizomes of Zingiber zerumbet and its anti-Staphylococcal activity. Lat. Am. Caribb. Bull. Med. Arom. Plants 2010, 9, 63-68.

16. Lee, J.H.; Choung, M.G. Determination of curcuminoid colouring principles in commercial foods by HPLC. Food Chem. 2011, 124, 1217-1222. [CrossRef] 
17. Paramasivam, M.; Poi, R.; Banerjee, H.; Bandyopadhyay, A. High-performancethin layer chromatographic method for quantitative determination of curcuminoids in Curcuma longa germplasm. Food Chem. 2009, 113, 640-644. [CrossRef]

18. Péret-Almeida, L.; Cherubino, A.P.F.; Alves, R.J.; Dufossé, L.; Glória, M.B.A. Separation and determination of the physico-chemical characteristics of curcumin, demethoxycurcumin and bisdemethoxycurcumin. Food Res. Int. 2005, 38, 1039-1044. [CrossRef]

19. Bos, R.; Windono, T.; Woerdenbag, H.J.; Boersma, Y.L.; Koulman, A.; Kayser, O. HPLC-photodiode array detection analysis of curcuminoids in Curcuma species indigenous to Indonesia. Phytochem. Anal. 2007, 18, 118-122. [CrossRef]

20. Balaji, S.; Chempakam, B. Toxicity prediction of compounds from turmeric (Curcuma longa L). Food Chem. Toxicol. 2010, 48, 2951-2959. [CrossRef]

21. Makabe, H.; Maru, N.; Kuwabara, A.; Kamo, T.; Hirota, M. Anti-inflammatory sesquiterpenes from Curcuma zedoaria. Nat. Prod. Res. 2006, 20, 680-685. [CrossRef]

22. Liao, Q.; Qian, Z.; Liu, R.; An, L.; Chen, X. Germacrone inhibits early stages of influenza virus infection. Antivir. Res. 2013, 100, 578-588. [CrossRef] [PubMed]

23. Lu, J.J.; Dang, Y.Y.; Huang, M.; Xu, W.S.; Chen, X.P.; Wang, Y.T. Anti-cancer properties of terpenoids isolated from Rhizoma curcumae-A review. J. Ethnopharmacol. 2012, 143, 406-411. [CrossRef] [PubMed]

(C) 2020 by the authors. Licensee MDPI, Basel, Switzerland. This article is an open access article distributed under the terms and conditions of the Creative Commons Attribution (CC BY) license (http://creativecommons.org/licenses/by/4.0/). 